\title{
Scalable Process for Application of Stabilized Lithium Metal Powder in Li-ion Batteries
}

Guo Ai ${ }^{\mathrm{a}, \mathrm{b}}$, Zhihui Wang ${ }^{\mathrm{a}}$, Hui Zhao ${ }^{\mathrm{a}}$, Wenfeng Mao ${ }^{\mathrm{a}}$, Yanbao Fu ${ }^{\mathrm{a}}, \mathrm{Ran}^{\mathrm{c}} \mathrm{i}^{\mathrm{c}}$, Yue Gao ${ }^{\mathrm{c}}$, Vincent Battaglia ${ }^{\mathrm{a}}$, Donghai Wang ${ }^{\mathrm{c}}$, Sergey Lopatin ${ }^{\mathrm{d}}$ and Gao Liu ${ }^{\mathrm{a}^{*}}$

${ }^{a}$ Energy Storage and Distributed Resources Division, Energy Technologies Area, Lawrence Berkeley National Laboratory, Berkeley, California 94720, United States

${ }^{b}$ Science and Technology on Reliability Physics and Application of Electronic Component Laboratory, No. 5 Electronic Research Institute of the Ministry of Industry and Information Technology, Guangzhou 510610, China

${ }^{c}$ Department of Mechanical and Nuclear Engineering, The Pennsylvania State University, University Park, Pennsylvania 16802,United States

${ }^{d}$ Applied Materials, Inc., Santa Clara, California 95054, United States.

*Corresponding author: Gao Liu; telephone: +1-510-486-7207; fax: +1-510-486-7303;

Email: gliu@1bl.gov (G. Liu)

\begin{abstract}
A simple solution processing method is developed to achieve a uniform and scalable stabilized lithium metal powder (SLMP) coating on a Li-ion negative electrode. A solvent and binder system for the SLMP coating is developed, including the selection of solvent, polymer binder, and optimization of polymer concentration. The optimized binder solution is a $1 \%$ concentration of polymer binder in xylene; a
\end{abstract} Page 1 of 16

(C) 2016. This manuscript version is made available under the Elsevier user license http://www.elsevier.com/open-access/userlicense/1.0/ 
mixture of poly(styrene-co-butadiene) rubber (SBR) and polystyrene (PS) is chosen as the polymer binder. Results show that long-sustained, uniformly dispersed SLMP suspension can be achieved with the optimized binder solution. The uniform SLMP coating can be achieved using a simple "doctor blade" coating method, and the resulting SLMP coating can be firmly glued on the anode surface. By using SLMP to prelithiate the negative electrode, improvements in electrochemical performances are demonstrated in both graphite/NMC and SiO/NMC full cells.

Keywords: Stabilized Lithium Metal Powder $\left(\operatorname{SLMP}^{\circledR}\right)$, Prelithiation, SiO, Full Cell 


\section{Introduction}

The explosion of technologies and products using Li-ion battery technology is causing manufacturers and consumers to demand higher and higher energy density and power output for portable electronic devices and electric vehicle applications [1-6]. Worldwide researchers are working to further improve Li-ion battery performance [7-11]. The new generation of Li-ion batteries with high-energy-density alloying anode materials [12, 13], such as silicon [14-18], germanium [19], and tin [14], have attracted significant attention. One limitation when using these materials is the high irreversible capacity loss (20\%-40\% depending on cell chemistry [20]), which results in the low coulombic efficiency (CE) in initial cycles [21]. In the effort to design a high-power battery, the reduction of active material particle size to nanoscale can help shorten the diffusion length of charge carriers, enhance the Li-ion diffusion coefficient, and therefore achieve faster reaction kinetics [22, 23]. However, using nano-size active material particles also leads to higher first-cycle irreversible capacity loss due to a larger reaction area for solid state electrolyte interface (SEI) formation. Many researchers have attempted to compensate for this capacity inefficiency, such as excess cathode material loading [24], increased lithium salt concentration [24], introducing lithium sacrificial salts [25], using lithium-rich cathode material [26] and the application of stabilized lithium metal powder (SLMP) [27-29].

SLMP is lithium metal particles coated with a very thin $\mathrm{Li}_{2} \mathrm{CO}_{3}$ layer $[30,31]$. This $\mathrm{Li}_{2} \mathrm{CO}_{3}$ protective coating enables the SLMP to be handled in a dry room atmosphere, which greatly increases its application. SLMP has a very high capacity of $3600 \mathrm{mAhg}^{-1}$ and can efficiently compensate for the first-cycle capacity loss with the prelithiation effect. Additionally, SLMP has several attractive uses. Firstly, the SLMP can prelithiate the anode electrode, especially the alloying anode [16-18, 32] or nano-scale anode, to compensate for the lithium loss in the cathode during SEI formation to achieve high energy-density and a high-power-density battery. Secondly, SLMP prelithiation can be used to take the place of the electrically charged formation process in Li-ion battery manufacturing. It is well known that the formation process is crucial in forming a good SEI layer, which is crucial in battery performance, but it is 
an energy and time consuming process. A high-quality SEI layer can be formed during SLMP prelithiation process, in which only SLMP loading, activation, and 48-hours of rest are required [33]. Thirdly, SLMP can also work as an independent source of lithium in some non-lithiated cathode materials with high specific capacities, [33] such as reduced graphene oxide/ $\mathrm{Fe}_{2} \mathrm{O}_{3}$ composite[34], $\mathrm{V}_{6} \mathrm{O}_{13}$ [35], $\mathrm{MnO}_{2}$ [36], and metal fluoride, $\mathrm{BiF}_{3}$ [29]. These materials are non-lithium-providing cathodes, and some of them can exhibit very high gravimetric and volumetric energy densities when they are coupled with SLMP prelithiated anodes in a Li-ion battery [33].

Although SLMP have already been proved to be cost-effective in Li-ion device applications [37], the handling and application of SLMP on the anode surface can be challenging. The $\mathrm{Li}_{2} \mathrm{CO}_{3}$ protective layer on the SLMP surface needs to be broken to expose Li metal and enable electrical contact between the lithium and the anode to achieve SLMP prelithiation. Therefore, pressure activation feasibility of SLMP should also be taken into account. In our previous work, efficient activation method of SLMP is studied $[18,33,38]$, and high first cycle coulombic efficiency as well as excellent long-term cycling performance in graphite/LiNi $\mathrm{Li}_{1 / 3} \mathrm{Co}_{1 / 3} \mathrm{Mn}_{1 / 3} \mathrm{O}_{2}$ (NMC) full cells. However, the need for SLMP research is not limited to the mechanism study and potentinal application prelithiation effect of SLMP — it is also needed to achieve a safer, less expensive and scalable process for the Li-ion batteries. Although the spray method has been shown to be effective for SLMP coating, a surfactant needs to be added to improve the SLMP dispersion, and side reactions may occur [39]. In this work, we develop a solution-processed slot dye coating method to achieve uniform and scalable SLMP coating on the anode surface. Also, polymer binders are introduced into the coating solution to assist and maintain uniform SLMP dispersion for prolonged processing time and to act as a binder to affix the SLMP on the anode surface. With the new approach, controllable and uniform SLMP coating can be formed on Li-ion anode surfaces and can be easily activated by applying calendaring pressure. The prelithiation effect of SLMP with this coating method is well demonstrated via the electrochemical performances in graphite/NMC and silicon monoxide (SiO)/NMC full cells. 


\section{Experimental Methodology}

\subsection{Materials and Electrode Fabrication}

For this research, a graphite anode mixture consisting of graphite (CGP-G8, Conoco Phillips), carbon black (Denka), and polyvinylidene fluoride (PVDF, Kureha America, Inc.) was used. The SiO anode consists of carbon-coated $\mathrm{SiO}$ (Hydro-Québec) and poly (9,9-dioctylfluorene-co-fluorenone-comethylbenzoic ester) (PFM, synthesized in our group). FMC Corporation produces the SLMP. Umicore provides the lithium nickel manganese cobalt oxide $\left(\mathrm{LiNi}_{1 / 3} \mathrm{Mn}_{1 / 3} \mathrm{Co}_{1 / 3} \mathrm{O}_{2}\right)$ cathode. N-Methyl-2pyrrolidone (NMP) (anhydrous, 99.5\%), chlorobenzene, toluene, xylene, and poly(styrene-co-butadiene) (SBR) are provided by Sigma Aldrich. Polystyrene (PS) (with molecular weight of 2,000,000) is purchased from Alfa Aesar.

The anode slurry is composed of $89 \mathrm{wt} \%$ CGP-G8 graphite powder, $8 \mathrm{wt} \%$ PVDF and $3 \mathrm{wt} \%$ acetylene black $(\mathrm{AB})$ and is homogenized with Polytron PT10-3 homogenizer before making the lamination. In this work, the graphite slurry was then coated with a Mitutoyo doctor blade on a Yoshimitsu Seiki vacuum coater. The current collector is an $18-\mu \mathrm{m}$-thick, battery-grade copper sheet. Typical mass loading of active material is $6.5 \mathrm{mgcm}^{-2}$. After NMP dried off, the electrode was further dried in a vacuum oven at $130^{\circ} \mathrm{C}$ for 16 hours before SLMP loading. The same mixing and coating procedure was applied for the $\mathrm{SiO}$ laminate, which is made with 95 wt \% $\mathrm{SiO}$ powder and 5\% PFM conductive binder in chlorobenzene. Typical mass loading of the $\mathrm{SiO}$ is $1.2 \mathrm{mgcm}^{-2}$. The cathode laminate for CGP-G8 graphite is $85 \mathrm{wt} \%$ $\mathrm{LiNi}_{1 / 3} \mathrm{Mn}_{1 / 3} \mathrm{Co}_{1 / 3} \mathrm{O}_{2}, 8 \mathrm{wt} \% \mathrm{PVDF}$, and $7 \mathrm{wt} \% \mathrm{AB}$. The cathode laminate for $\mathrm{SiO}$ is made by Argonne National Lab with 90 wt \% NCM 622, 5 wt \% PVDF (Solvay 5130) and 5 wt \% C45 (Timcal).

\subsection{SLMP Coating Procedure}

The binder solution is made by dissolving SBR and PS well in xylene using different weight ratios. Then SLMP is added and well dispersed in the binder solution to form SLMP suspension with SLMP weight ratio of $0.5 \%$ (for graphite) and $2.0 \%$ (for $\mathrm{SiO}$ ). The loading amount can be controlled by varying the 
amount of SLMP in solution. Then the SLMP suspension is coated on the anode laminate with the doctor blade method. The gap for the doctor blade is also tunable to control the SLMP loading on the laminate surface. After the xylene is dried off, the electrode with SLMP coating is calendared with a rolling press (EQ-MR 100A from MTI Corporation) to activate the SLMP. Then the laminate is punched for cell assembling.

\subsection{Cell Assembly and Testing}

The prelithiation effect of SLMP is tested by making a 2325-type coin cell (parts from National Research Council, Canada). The SLMP-loaded cells are assembled in an argon-filled glove box with an oxygen mixing ratio less than $0.2 \mathrm{ppm}$. The pressure-activated SLMP-graphite and SLMP-SiO are used as anodes. The counter electrodes for the half-cell are a lithium metal disk of 11/16" OD (Li foil from FMC-Lithium Company). The electrolyte for the graphite half and full cells is composed of a $1 \mathrm{M} \mathrm{LiPF}_{6}$ solution in ethyl carbonate (EC) and diethyl carbonate (DEC) with volume ratio of 1:1, purchased from BASF. The electrolyte for $\mathrm{SiO}$ half and full cell is composed of a $1 \mathrm{M} \mathrm{LiPF}_{6}$ solution in ethyl carbonate (EC) and diethyl carbonate (DEC) with a weight ratio of 3:7 containing $30 \mathrm{wt} \%$ fluoroethylene carbonate (FEC) purchased from BASF. A Celgard 2400 separator is used. Galvanostatic cycling tests were performed on a Maccor series 4000 cell tester. For graphite and $\mathrm{SiO}$ half cells, the voltage window was $0.01-1.0 \mathrm{~V}$. The cells containing pressure-activated SLMP were rested for 2 days at open circuit before cycling to let prelithiation reach equilibrium. For the full cell, the ratio of area capacity for the cathode and anode is 1:1. The capacity of the cell is calculated based on the cathode.

\subsection{Material Characterization}

Morphology of the electrode surface is characterized with a JSM-7500F scanning electron microscope at the National Center for Electron Microscopy (NCEM) of Molecular Foundry at Lawrence Berkeley National Laboratory. The viscosity is tested via a DV-E viscometer manufactured by Brookfield. The test is performed at room temperature $\left(23^{\circ} \mathrm{C}\right)$. 


\section{Results and Discussion}

\subsection{Choice of Solvent for Solution Processing Method}

In the solution processing method, the compatibility between solvent and SLMP is important. Results of previous investigations of SLMP stability in different solvents [29] have shown that SLMP is not compatible with some polar solvents such as 1-methyl-2-pyrrolidinone (NMP) [29]. Only hydrocarbon solvents are stable with lithium metal such as hexane, toluene, and xylene. As for the selection of binders for the SLMP slurry, poly(styrene-co-butadiene) (SBR) has already been successfully used in previous SLMP work. Therefore, SBR was selected as binder for the SLMP slurry first. Both polymer binders can dissolve in the toluene and xylene to form a uniform solution. The ability of binder solution to maintain uniformity of SLMP suspension for long periods of time is very important. Therefore, a solvent with higher viscosity is preferred. The viscosity of toluene and xylene are $0.55 \mathrm{cP}$ and $0.61 \mathrm{cP}$ at $25^{\circ} \mathrm{C}$, respectively $[40,41]$. This indicates that xylene should be better at sustaining SLMP slurry than toluene. However, neither pure toluene nor xylene is viscous enough to maintain the SLMP dispersion in the slurry. As indicated in Fig. 1, the particles tend to float onto the surface of the solvent because of the extremely small density of SLMP and low viscosity of the solution. What's more, the SLMP will fly away after the solvent is evaporated because no attachment force exists between the SLMP and the laminate surface if no polymer binder is included. Therefore, in this study, polymer binder is introduced into the solution processing method and its effect on the SLMP coating is further investigated.

\subsection{Concentration and Viscosity of Binder Solution for Sustained SLMP Suspension}

By introducing the polymer into the solution-processing method for the SLMP coating, the viscosity of the solution can be greatly increased. Therefore a well-dispersed SLMP suspension can maintain uniform dispersion for long handling times. The introduced polymers can act as binders to affix the SLMP on the anode surface after the solvent has evaporated. SBR and PS are selected as the binders in this research. SBR has a strong cohesive ability that can provide good attachment between the SLMP and anode surface 
[38]. The characteristics of the polymer solution with different SBR concentration $(0 \%, 0.5 \%, 1 \%, 3 \%$ and $5 \%$ ) on SLMP coating are studied. The viscosity is tested to be $1.02 \mathrm{cP}, 2.52 \mathrm{cP}, 15.00 \mathrm{cP}$, and $36.90 \mathrm{cP}$ for $0.5 \%, 1 \%, 3 \%$, and $5 \%$ SBR solution, respectively. The same amounts of SLMP are dispersed in each SBR solution to demonstrate the sustained ability of each binder solution for SLMP slurry. Photo images are taken after the slurries are well mixed and rested for different time durations: 0 second, 30 seconds, and 120 seconds, respectively. As shown in Fig. 2, the addition of polymer binder can effectively increase the viscosity of the solution and maintain the uniform distribution of the SLMP in the slurry. It also indicates that the higher concentration of polymer binder solution, the better sustainability of SLMP distribution in the slurry.

The morphology and distribution of the SLMP coating with different SBR concentration are studied with SEM, Fig. 2 (d-o). Significant difference is observed among them both at low- and high-magnification images. For the coating with $0.5 \%$ SBR, very little SLMP is coated on the anode surface, Fig. 2 (d-f). This is because the viscosity for the $0.5 \%$ SBR solution is too low $(1.02 \mathrm{cP})$ and the difference in density between lithium and xylene is large (the density for lithium is $0.534 \mathrm{gcm}^{-3}$, while the density for xylene is $\left.0.87 \mathrm{gcm}^{-3}\right)$. In this scenario, most of the SLMP tend to float on the surface of the coating suspension during the coating process and are mostly removed by the doctor blade. When the SBR concentration increases to $1 \%$, viscosity increases to $2.52 \mathrm{cP}$; SLMP distribution can be well sustained in the slurry for 120 seconds, Fig. 2 (a-c), and uniform SLMP coating can be achieved on the anode surface, Fig. 2 (g-i). Further increasing the SBR concentration to $3 \%$ and 5\%, the SLMP coating can be as uniform, but the amount of SLMP coated on the laminate surface does not seem to increase much compared to the coating performed with $1 \%$ SBR solution, Fig. 2 (j-o). These results show that the ability to sustain SLMP slurry increases with higher viscosity and uniform SLMP coating can be achieved when the viscosity of the binder solution is above $2.5 \mathrm{cP}$. 
For the following discussion, binder concentration is set to be $1 \%$ with the above optimization. Further efforts will be focused on achieving uniform coating and easy pressure activation. Minor adjustments could be made when other polymer binders are used.

\subsection{Choices of Binder Species for High-Efficiency Pressure/Calendar Activation}

Another important issue that needs to be addressed for the application of SLMP is the activation process. An easy-pressure activation method was developed in previous studies [33]. But when the polymer binder is introduced in the processing method, activation feasibility and efficiency have to be re-evaluated. When examining the SEM images of SLMP with 5\% SBR binder, a polymer shell is observed on the SLMP surface, Fig. 2 (1). In comparison, this polymer shell is not observed at $1 \%$ binder solution, Fig. 2 (f, i), and minimal shell is observed on SLMP coated with 3\% SBR, Fig. $2(\mathrm{k}, 1)$. The primary question for process development is if this thin polymer shell has any negative effect on the pressure activation process. Therefore, the SEM morphology of SLMP-coated anode before and after adding electrolyte is studied in Fig. 3, and 3\% concentration of the polymer binder is used to demonstrate the effect of this polymer shell.

By applying adequate pressure to the SLMP-coated anode, most of the $\mathrm{Li}_{2} \mathrm{CO}_{3}$ coating of SLMP can be smashed, as shown in SEM images in Fig. 3 (a, b). After immersing the pressure-activated, SLMP-coated anode in the electrolyte for 48 hours, the prelithiated anode is observed via SEM again. The smashed SLMP particles disappear from view in Fig. 3 (c). But, taking a closer look using a high-magnification SEM image, a few un-reacted SLMP particles can be found, as shown in Fig. 3 (d). The oblate morphology suggests that these particles have been exposed to pressure, but that the lithium is not directly electrically contacted with the electrode: they did not react with the anode and electrolyte. Because SBR is well known for high elasticity, the shell is not efficiently broken under pressure. The SBR shell on the SLMP surface prevents the electrical contact between the lithium core, anode, and electrolyte and leads to insufficient pressure SLMP activation. 
There are two ways to eliminate this inefficient activation caused by the polymer binder shell. First, by lowering the binder concentration, there is less chance to form a complete shell. But lower binder concentration may result in other issues, such as non-uniform SLMP dispersion, poor sustainability, and poor attachment on the anode surface. Second, choosing a polymer binder with less elasticity as alternative is important for this application. The commercially available polystyrene (PS) is introduced. The transition temperature (Tg) is $95^{\circ} \mathrm{C}$ for PS and $-65^{\circ} \mathrm{C}$ for SBR, which indicates that PS is more brittle and easier to break compared to SBR, largely helping the pressure activation process. Additionally, PS with high molecular weight was easily obtained, so a higher viscosity PS solution can be obtained than SBR when the concentration is the same.

Three sets of binder solution (1\% PS, $1 \%$ SBR, and $0.5 \%$ PS with $0.5 \%$ SBR) are introduced to optimize the binder composition for easier pressure activation. The SEM images of SLMP loading on graphite surface before, after calendaring, and after immersing in electrolyte for 48 hours with $1 \%$ PS, $1 \%$ SBR, and $0.5 \%$ PS with $0.5 \%$ SBR binder solutions are shown in Fig. 4. Uniform SLMP coating is observed for SLMP coating with $1 \%$ SBR and $0.5 \%$ PS with $0.5 \%$ SBR binder solutions, Fig. 4(b, c). The uniformity of SLMP coating will be discussed in the next section. The SLMP is smashed on the graphite surface after calendaring and the anodes have similar morphology with different binder solutions, Fig. 4 (d-f). After the calendared electrodes are immersed in the electrolyte for 48 hours, all the smashed SLMP particles disappear from SEM view. Almost none of the residual SLMP, found in Fig. 3 (d), is observed under the high-magnification SEM observation of SLMP coating with $1 \%$ PS solution and $0.5 \%$ PS with $0.5 \%$ SBR solution, Fig. 4 (g, i). Only two residual SLMP particles are observed in Fig. 4 (h). This indicates that the optimized binder solutions, which partially or totally use PS as polymer binder, have positive effect in achieving high-pressure activation efficiency.

\subsection{Uniformity of SLMP Coating with Optimized Binder Solution}


To investigate the sustainability of the three binder solutions, SLMP slurries are made with the 1\% PS, $1 \%$ SBR, and $0.5 \%$ PS with $0.5 \%$ SBR solutions, respectively. The viscosity of the binder solutions with $1 \% \mathrm{PS}, 1 \% \mathrm{SBR}$, and $0.5 \% \mathrm{PS}$ with $0.5 \% \mathrm{SBR}$ is $4.83 \mathrm{cP}, 2.52 \mathrm{cP}$, and $3.60 \mathrm{cP}$, respectively. This further suggests that PS with high molecular weight can lead to higher viscosity. Photo images of the SLMP suspension are taken after different time durations for 0 second, 30 seconds, and 120 seconds in Fig. 5 (a, $\mathrm{b}$, and c). These images show that, in all three solvents, SLMP are uniformly distributed for longer than 120 seconds. The phase separation of SLMP slurry begins to be observable after 5-6 minutes. Since this work is aimed at designing a slurry composition for a continuous coating procedure performed right after the slurry is mixed, it indicates that this composition of this polymer binder offers enough time to process the homogenously dispersed SLMP suspension with the continuous coating procedure.

The doctor blade method is performed for coating SLMP slurries in binder solution (1\% PS, $1 \%$ SBR, and $0.5 \%$ PS with $0.5 \%$ SBR in xylene). Photo images of SLMP-coated graphite anodes with different binder solutions exhibit their abilities in achieving uniform SLMP coating and good SLMP attachment. In the three binder solutions, the sustainability of SLMP slurries is comparable and the appearances of SLMP coatings are similar before solvent evaporation. The SLMP coating with $1 \%$ PS binder solution exhibits poor attachment on the electrode surface. Most of SLMP particles tend to float to the edge instead of attaching on the anode surface after the solvent evaporates, Fig. 5(d). However, the $1 \%$ SBR and $0.5 \%$ PS with $0.5 \%$ SBR binder solutions show that a uniform SLMP coating and good attachment of SLMP on the anode surface can be achieved after the solvent evaporates. The reason for this difference is that the transition temperature ( $\mathrm{Tg}$ ) is $95^{\circ} \mathrm{C}$ for PS and $-65^{\circ} \mathrm{C}$ for SBR. Glass transition temperature is the temperature below which the polymers are in glass phase and the polymer structure is rigid. Therefore, at room temperature $\left(25^{\circ} \mathrm{C}\right)$, the PS is in a brittle glassy state, and cannot act as soft glue; but the SBR is in soft, flexible rubbery state, and can act as an effective glue to affix SLMP on the anode surface. The effective attachment of SLMP with SBR binder is partly due to the high flexibility of the SBR chemical structure. In the case of $1 \%$ PS without SBR, poor SLMP attachment is caused by the rigid PS structure. 
To overcome this problem, both polymers are used, combining the PS and SBR into a mixed-binder solution. The flexible SBR can help to achieve a good SLMP attachment, while the rigid PS can help to achieve much easier pressure activation. Therefore, with the overall consideration of achieving long sustained SLMP slurry, uniform SLMP coating, good SLMP adhesion, and easy activation, the best composition of binders and solvent combination is $0.5 \%$ PS with $0.5 \%$ SBR binder in xylene solution for the processing method. This superb coating effect is shown in Fig. 5 (f).

\subsection{Prelithiation Effect in Graphite/NMC Full Cell}

Two types of cell chemistry have been used to demonstrate the prelithiation effect of SLMP coated with this solution processing method: graphite/NMC full cell and high-energy-density SiO/NMC full cell. The prelithiation effects of SLMP on graphite/NMC full cells and graphite half cells are plotted in Fig. 6. Fig. 6 (a) is the first-cycle voltage profile for the graphite/NMC full cells with and without SLMP prelithiation. In the voltage region below $3.5 \mathrm{~V}$, the cell without SLMP prelithiation shows charge capacity that corresponds to the lithiation of the graphite anode with the SEI formation in the first cycle. In cells with SLMP prelithiation, SEI has been formed in the prelithiation process before cycling and the graphite has already been partially lithiated; open-circuit voltage starts from $3.5 \mathrm{~V}$ and almost no capacity is shown below $3.5 \mathrm{~V}$. The lithium ion loss in the full cell during SEI formation can be compensated by the addition of SLMP so that the first-cycle columbic efficiency is increased to $87.8 \%$, which is higher than the $82.35 \%$ for graphite/NMC cells without SLMP prelithiation (as calculated from Fig. 6 (b)). Also, the

cycle stability of the graphite/NMC full cell can be greatly improved with the SLMP prelithiation. This indicates that the failure mechanism of irreversible lithium loss in the full cell can be partially compensated by the addition of the extra lithium ion from SLMP. The prelithiation effect of SLMP on the anode can also be observed in the voltage profile of the graphite half cell, Fig. 6 (c). The voltage plateau at $0.7-0.8 \mathrm{~V}$ in the half cell without SLMP prelithiation indicates the SEI formation at the beginning of lithiation process. The starting voltage of SLMP prelithiated half cell has already reached lower than 0.3 V, Fig. 6 (c), which further proves that the SEI formation has been achieved in the SLMP prelithiation 
process. Cycle performances of graphite and the NMC half cells are shown in Fig. 6 (d) and (f), respectively, along with the voltage profile of the NMC half cell for reference, Fig. 6 (e).

\subsection{Prelithiation Effect in SiO/NMC Full Cell}

The prelithiation of SLMP becomes crucially important in other high-energy-density anodes, such as Si or $\mathrm{SiO}$, etc., because of their up to $40 \%-50 \%$ first cycle coulombic loss. The prelithiation effect of SLMP is demonstrated by SiO/NMC full cell with the solution processing method of SLMP coating, Fig. 7. The $\mathrm{SiO}$ anode is composed of $\mathrm{SiO}$ nanoparticle (95 wt.\%) with conductive binder (PFM, 5 wt.\%). Significant improvement is observed in the SLMP prelithiated full cells, Fig.7 (b). The first cycle CE increases from $56.78 \%$ (without SLMP prelithiation) to $88.12 \%$ (with SLMP prelithiation) with this solution processing method. This is because that, in the full cell design, the lithium ion in the cathode is accurately calculated to match the capacity of the anode and not much excess lithium ion exists. The formation of SEI in the first few cycle will consume most of the excess lithium ion and the long term cycle ability will suffer due to the lithium ion loss. Furthermore, lithium ion consumption during the SEI formation is much higher in the $\mathrm{SiO}$ anode than in the graphite anode. Without the prelithiation of SLMP, the SiO/NMC full cell can only start with a capacity of $\sim 110 \mathrm{mAhg}^{-1}$ and drop to $\sim 80 \mathrm{mAhg}^{-1}$ after 100 cycles (based on cathode weight). With the prelithiation of SLMP, the SiO/NMC full cell can maintain a reversible capacity of 130 $\mathrm{mAhg}^{-1}$ for more than one hundred cycles. With the coating of SLMP on the anode of the full cell, a wellcalculated amount of lithium ion is added into the system, compensating for lithium ion loss during SEI formation. In this way, a significant improvement in cycle ability can be observed in the SiO/NMC full cell.

\section{Conclusion}

This work introduces a simple solution processing method to achieve large-area, uniform SLMP coating on a well-made anode surface for the prelithiation of lithium-ion batteries. By optimizing the composition of the binder solution, the SLMP can sustain uniform distribution in the slurry to carry out the coating 
process. By adding polymer binders, the SLMP can be fixed on the anode surface for easy transportation before activation. Considering sustainability and coating performance, the SLMP adhesion, and the ease of activation, the best composition of binders and solvent combination is $0.5 \%$ PS with $0.5 \%$ SBR binder in xylene solution for the processing method-resulting in a superb coating effect. The prelithiation effect with this method is applied both in graphite half cells, graphite/NMC full cells, SiO half cells, and $\mathrm{SiO} / \mathrm{NMC}$ full cells with improvements in cycle performance and higher first-cycle coulombic efficiency than their corresponding cells without SLMP prelithiation. These results demonstrate that this method is both effective and has wide applicability in different anode systems with different cell chemistries.

\section{Acknowledgements}

This work is funded by the Assistant Secretary for Energy Efficiency, Office of Vehicle Technologies of the U.S. Department of Energy (U.S. DOE) under the Advanced Battery Materials Research (BMR) program, along with the National Center for Electron Microscopy of the Molecular Foundry and the Advanced Light Source at the Lawrence Berkeley National Laboratory, which are supported by the U.S. Department of Energy under Contract \# DE-AC02-05 CH11231. Guo Ai is supported by the funding from the Distinguished Young Scientist Program of Guangdong Province (2015A030306002) and the China Scholarship Council. Ziyan Zheng (University of California, Berkeley) provided comments and modifications to the manuscript.

\section{References}

[1] J.B. Goodenough, Y. Kim, Chemistry of Materials, 22 (2010) 587-603.

[2] F.T. Wagner, B. Lakshmanan, M.F. Mathias, The Journal of Physical Chemistry Letters, 1 (2010) 2204-2219.

[3] J.M. Tarascon, M. Armand, Nature, 414 (2001) 359-367.

[4] Y. Dai, L. Cai, R.E. White, Journal of The Electrochemical Society, 160 (2013) A182-A190.

[5] Y. Dai, L. Cai, R.E. White, Journal of The Electrochemical Society, 161 (2014) E3348-E3356. 
[6] Y. Dai, L. Cai, R.E. White, Journal of Power Sources, 247 (2014) 365-376.

[7] W.-f. Mao, Y. Ma, S.-k. Liu, Z.-y. Tang, Y.-b. Fu, Electrochimica Acta, 147 (2014) 498-505.

[8] W.-f. Mao, J. Yan, H. Xie, Z.-y. Tang, Q. Xu, Journal of Power Sources, 237 (2013) 167-171.

[9] G. Ai, Y. Dai, Y. Ye, W. Mao, Z. Wang, H. Zhao, Y. Chen, J. Zhu, Y. Fu, V. Battaglia, J. Guo, V. Srinivasan, G. Liu, Nano Energy, 16 (2015) 28-37.

[10] W.-f. Mao, Y.-b. Fu, H. Zhao, G. Ai, Y.-l. Dai, D. Meng, X.-h. Zhang, D. Qu, G. Liu, V.S. Battaglia, Z.-y. Tang, ACS Applied Materials \& Interfaces, 7 (2015) 12057-12066.

[11] Y. Dai, V. Srinivasan, Journal of The Electrochemical Society, 163 (2015) A406-A416.

[12] L. Ji, Z. Lin, M. Alcoutlabi, X. Zhang, Energy \& Environmental Science, 4 (2011) 2682-2699.

[13] X.-L. Wu, Y.-G. Guo, L.-J. Wan, Chemistry - An Asian Journal, 8 (2013) 1948-1958.

[14] U. Kasavajjula, C. Wang, A.J. Appleby, Journal of Power Sources, 163 (2007) 1003-1039.

[15] Y. Bai, Y. Tang, Z. Wang, Z. Jia, F. Wu, C. Wu, G. Liu, Solid State Ionics, 272 (2015) 24-29.

[16] G. Liu, S. Xun, N. Vukmirovic, X. Song, P. Olalde-Velasco, H. Zheng, V.S. Battaglia, L. Wang, W. Yang, Advanced Materials, 23 (2011) 4679-4683.

[17] S.-J. Park, H. Zhao, G. Ai, C. Wang, X. Song, N. Yuca, V.S. Battaglia, W. Yang, G. Liu, Journal of the American Chemical Society, 137 (2015) 2565-2571.

[18] H. Zhao, Z. Wang, P. Lu, M. Jiang, F. Shi, X. Song, Z. Zheng, X. Zhou, Y. Fu, G. Abdelbast, X. Xiao, Z. Liu, V.S. Battaglia, K. Zaghib, G. Liu, Nano Letters, 14 (2014) 6704-6710.

[19] D.D. Vaughn Ii, R.E. Schaak, Chemical Society Reviews, 42 (2013) 2861-2879.

[20] M.W. Forney, M.J. Ganter, J.W. Staub, R.D. Ridgley, B.J. Landi, Nano Letters, 13 (2013) 41584163.

[21] V.A. Agubra, J.W. Fergus, Journal of Power Sources, 268 (2014) 153-162.

[22] X. Su, Q. Wu, J. Li, X. Xiao, A. Lott, W. Lu, B.W. Sheldon, J. Wu, Advanced Energy Materials, 4 (2014) 1300882.

[23] Y. Wang, H. Li, P. He, E. Hosono, H. Zhou, Nanoscale, 2 (2010) 1294-1305.

[24] J. Christensen, J. Newman, Journal of The Electrochemical Society, 152 (2005) A818-A829. 
[25] D. Shanmukaraj, S. Grugeon, S. Laruelle, G. Douglade, J.M. Tarascon, M. Armand, Electrochemistry Communications, 12 (2010) 1344-1347.

[26] A. Ito, D. Li, Y. Sato, M. Arao, M. Watanabe, M. Hatano, H. Horie, Y. Ohsawa, Journal of Power Sources, 195 (2010) 567-573.

[27] C.R. Jarvis, M.J. Lain, Y. Gao, M. Yakovleva, Journal of Power Sources, 146 (2005) 331-334.

[28] C.R. Jarvis, M.J. Lain, M.V. Yakovleva, Y. Gao, Journal of Power Sources, 162 (2006) 800-802.

[29] B.B. Fitch, M. Yakovleva, Y. Li, I. Plitz, A. Skrzypczak, F. Badway, G.G. Amatucci, Y. Gao, ECS Transactions, 3 (2007) 15-22.

[30] J.T. Vaughey, G. Liu, J.-G. Zhang, MRS Bulletin, 39 (2014) 429-435.

[31] Y. Li, B. Fitch, Electrochemistry Communications, 13 (2011) 664-667.

[32] H. Zhao, N. Yuca, Z. Zheng, Y. Fu, V.S. Battaglia, G. Abdelbast, K. Zaghib, G. Liu, ACS Applied Materials \& Interfaces, 7 (2015) 862-866.

[33] Z. Wang, Y. Fu, Z. Zhang, S. Yuan, K. Amine, V. Battaglia, G. Liu, Journal of Power Sources, 260 (2014) 57-61.

[34] X. Zhu, Y. Zhu, S. Murali, M.D. Stoller, R.S. Ruoff, ACS Nano, 5 (2011) 3333-3338.

[35] D.W. Murphy, P.A. Christian, F.J. DiSalvo, J.N. Carides, J.V. Waszczak, Journal of The Electrochemical Society, 128 (1981) 2053-2060.

[36] K.-W. Nam, S.-B. Ma, W.-S. Yoon, X.-Q. Yang, K.-B. Kim, Electrochemistry Communications, 11 (2009) 1166-1169.

[37] W.J. Cao, J. Shih, J.P. Zheng, T. Doung, Journal of Power Sources, 257 (2014) 388-393.

[38] L. Wang, Y. Fu, V.S. Battaglia, G. Liu, RSC Advances, 3 (2013) 15022-15027.

[39] F. Cassel, D. Chua, M. Lane, M. Yakovleva, Y. Gao, G. Au, ECS Transactions, 11 (2008) 157-166.

[40] F.J.V. Santos, C.A. Nieto de Castro, J.H. Dymond, N.K. Dalaouti, M.J. Assael, A. Nagashima, Journal of Physical and Chemical Reference Data, 35 (2006) 1-8.

[41] S. Singh, B.P.S. Sethi, R.C. Katyal, V.K. Rattan, Journal of Chemical \& Engineering Data, 50 (2005) 125-127. 


\section{(a) 0 second}

\section{(b) 30 second}

Toluene Xylene
Toluene Xylene

\section{(c)120 second}

Toluene Xylene
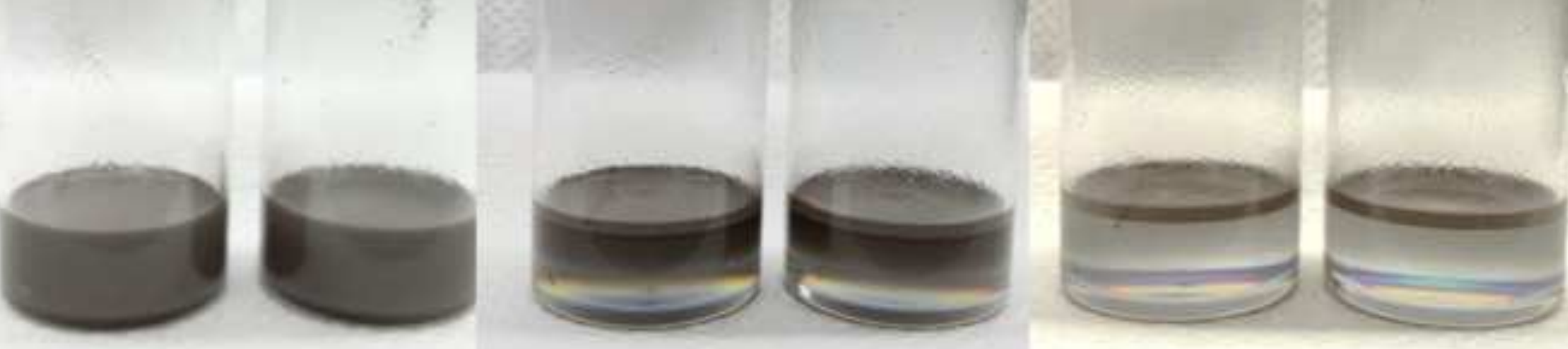


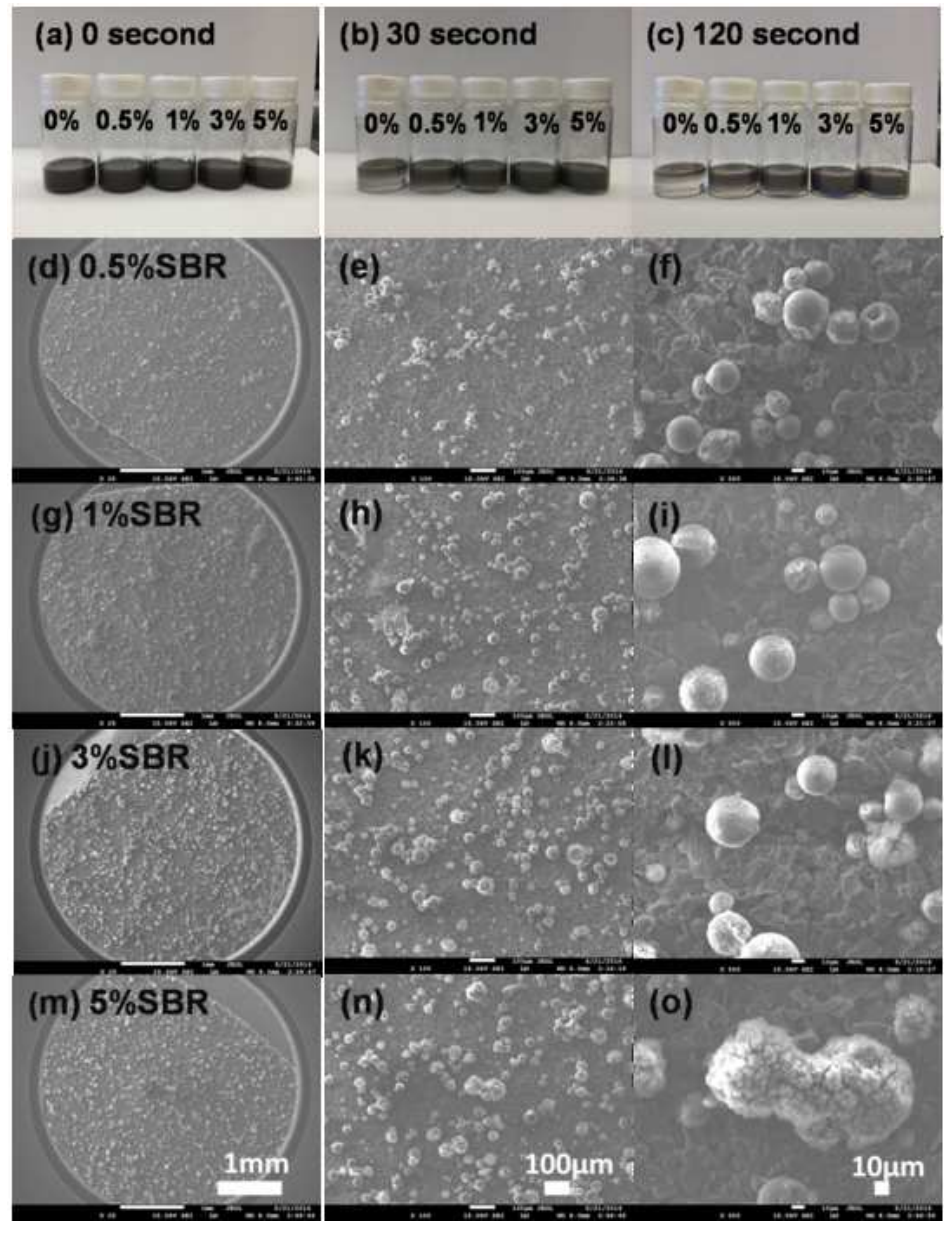

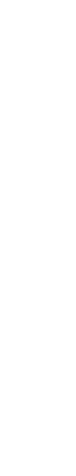

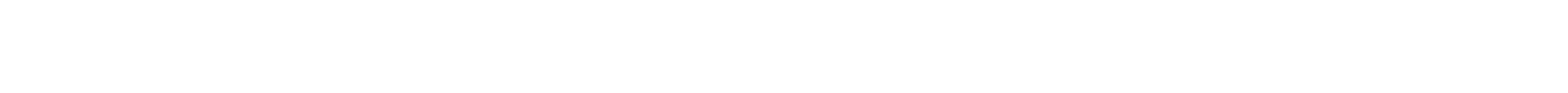




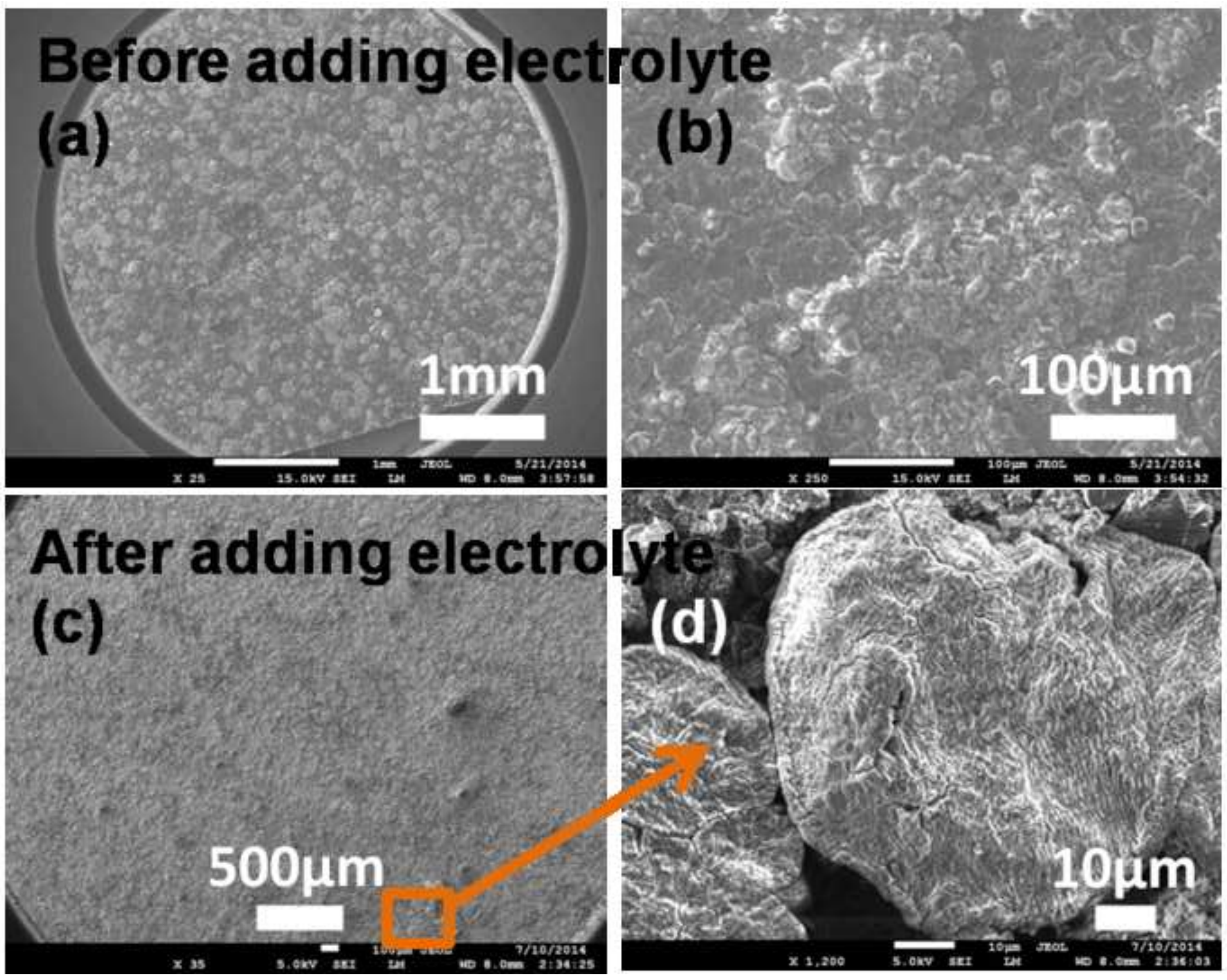


(a) $10 \% \mathrm{ps}$

Before calendaring

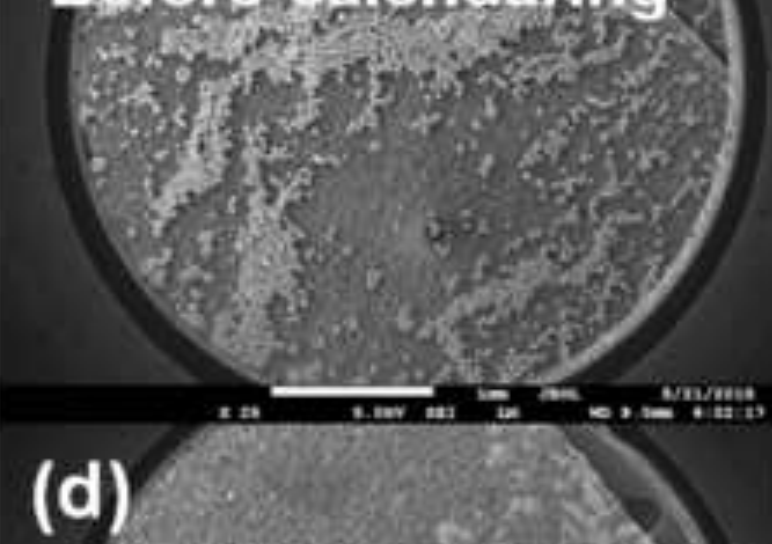

After calendaring

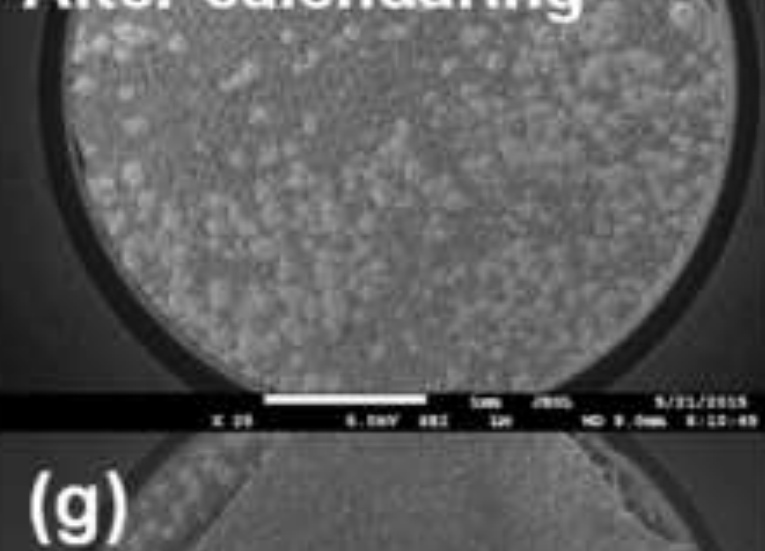

(b) $1 \%$ SBR

(e)

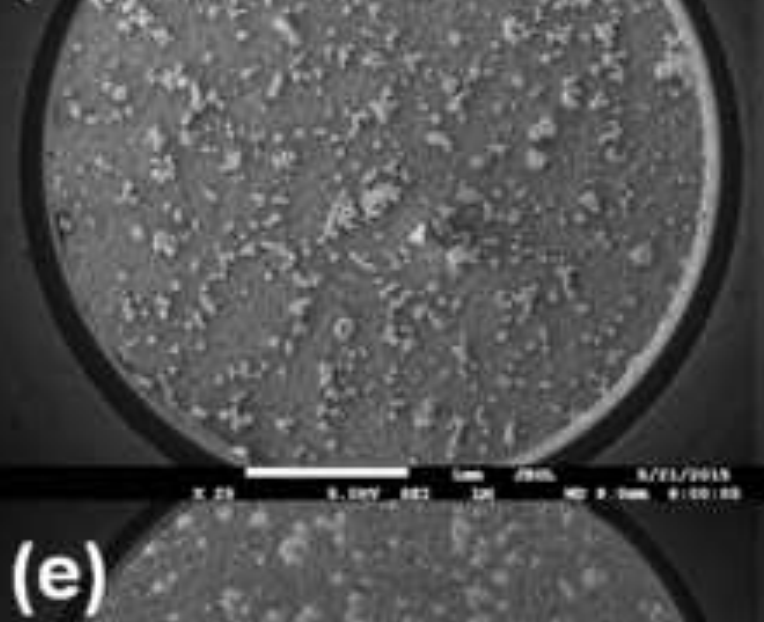

(f)
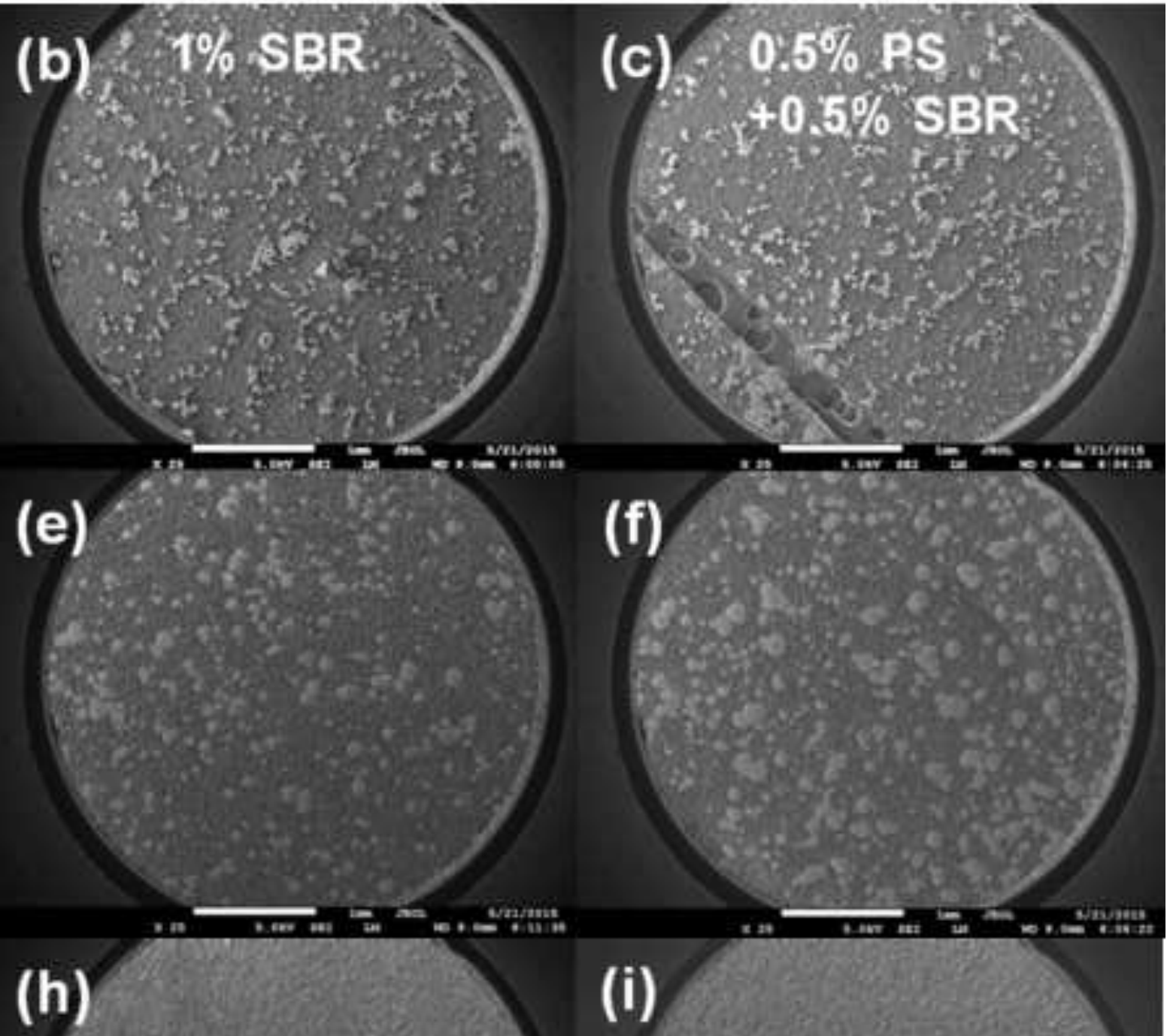

(i)

After immersing in electrolyte for 48 hours
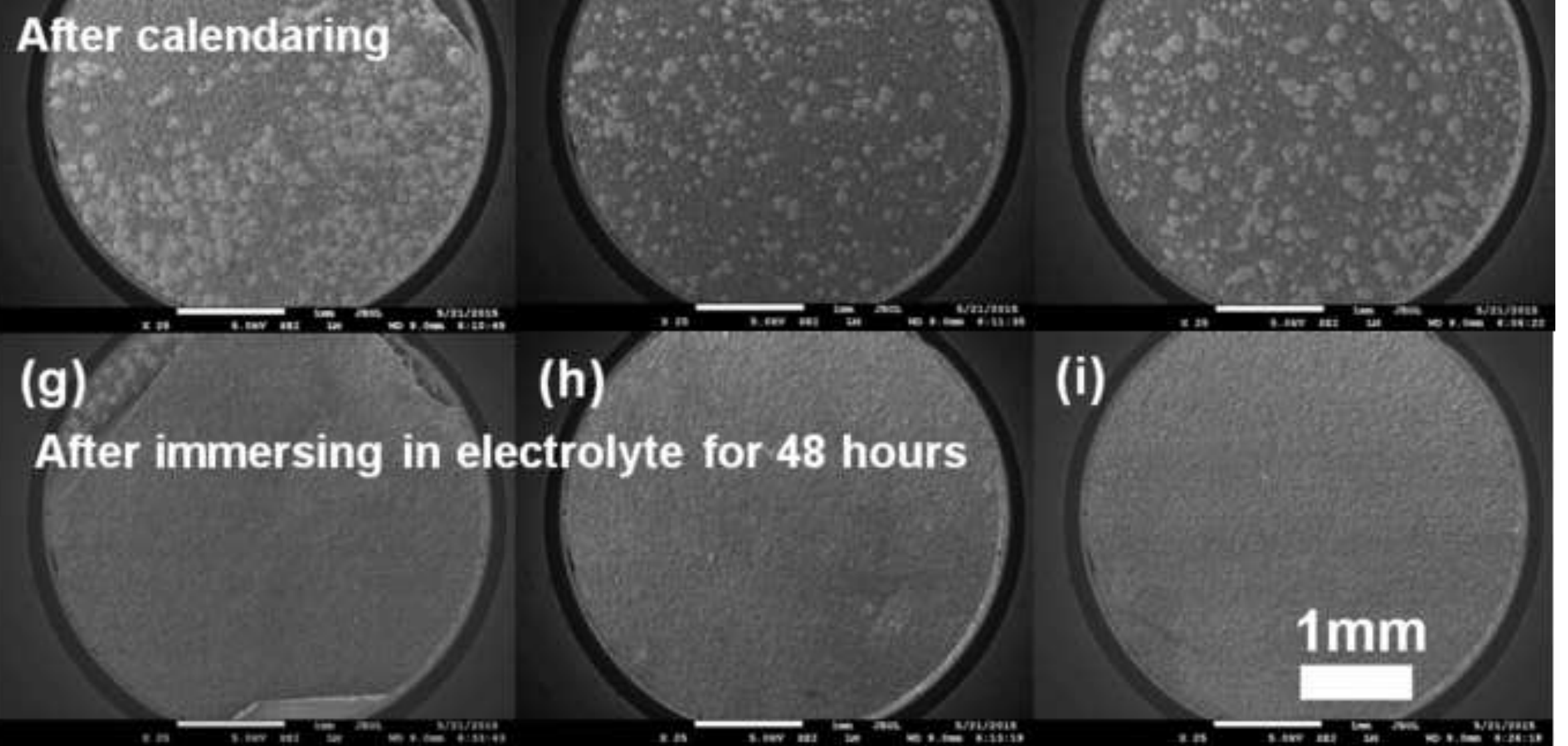


\section{(a) 0 second \\ (b) $\mathbf{3 0}$ second \\ (c) 120 second}

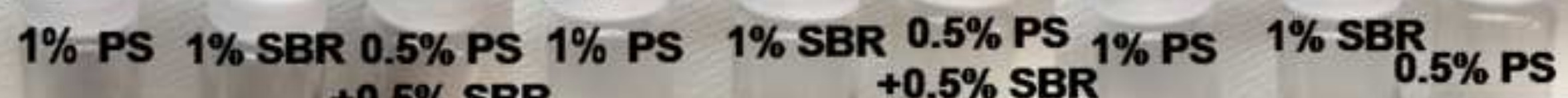

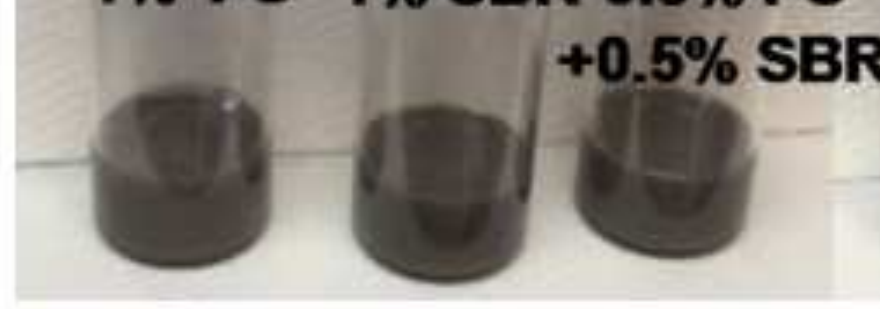

(d) $1 \%$ PS

(e) $1 \%$ SBR

$2 \mathrm{~cm}$

\section{$2 \mathrm{~cm}$}

(f) $0.5 \%$ PS $+0.5 \% \mathrm{SBR}$

\section{$5 \mathrm{~cm}$}


(a)

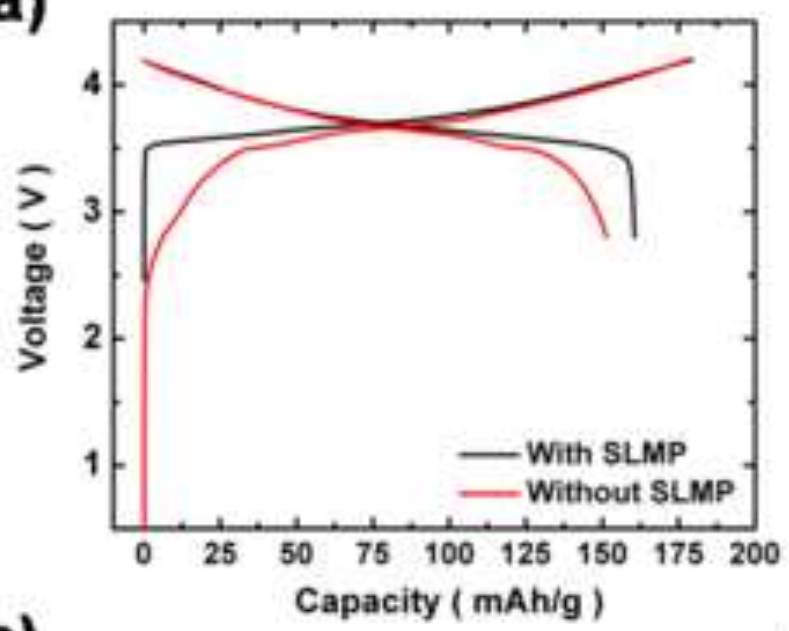

(c)

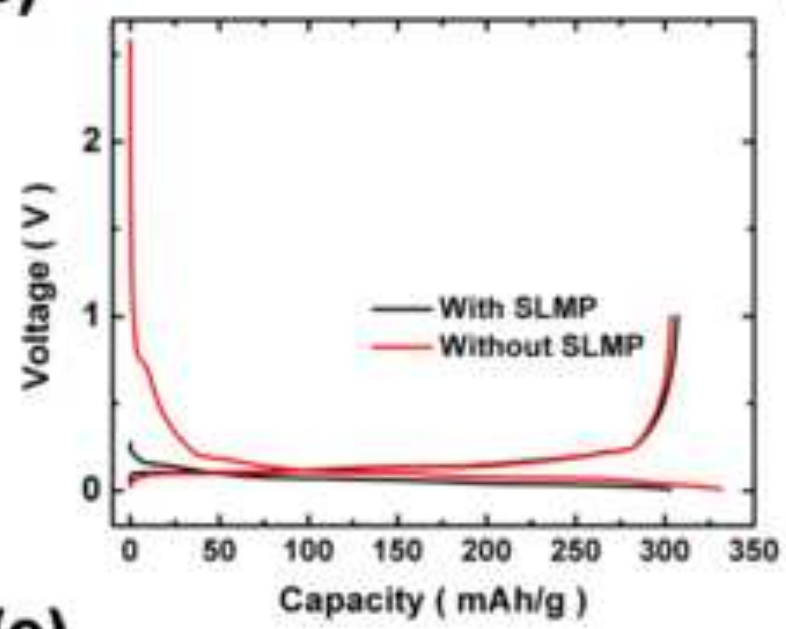

(e)

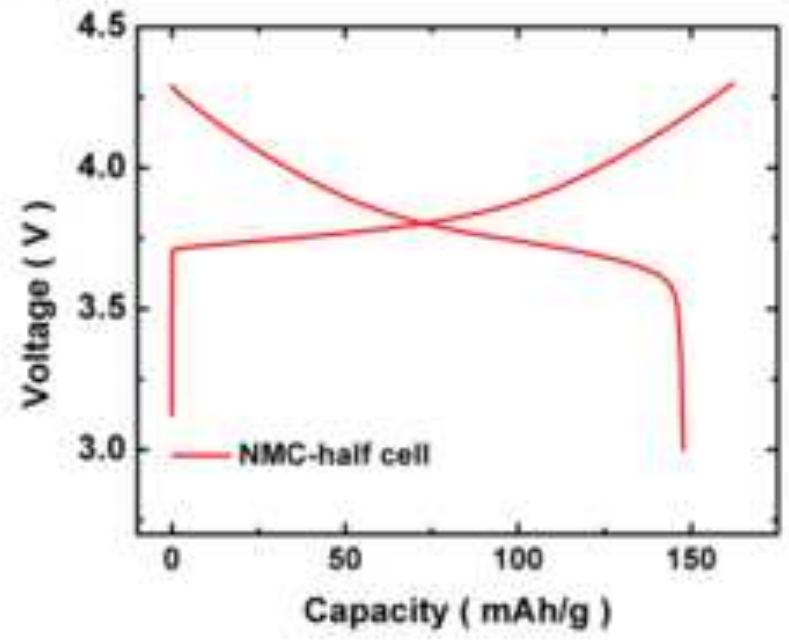

(b)

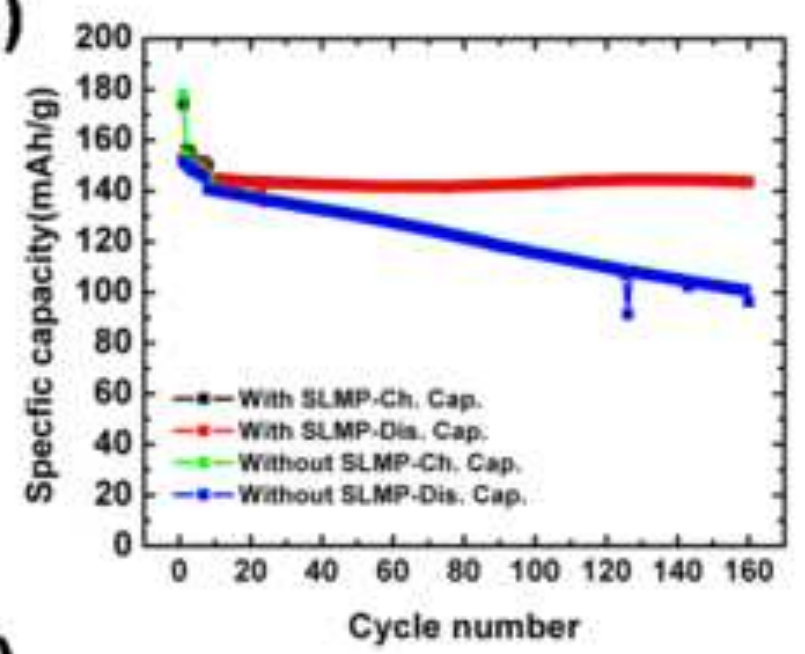

(d)

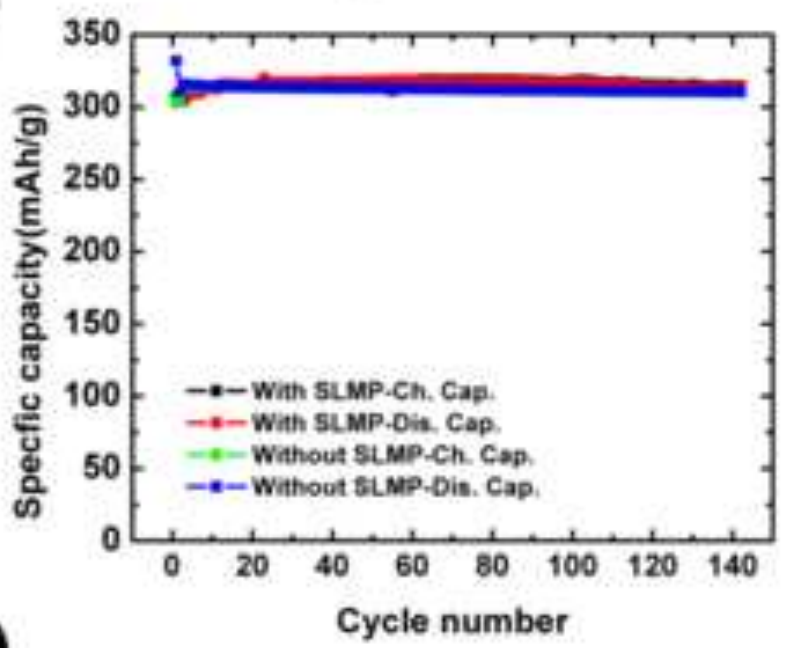

(f)

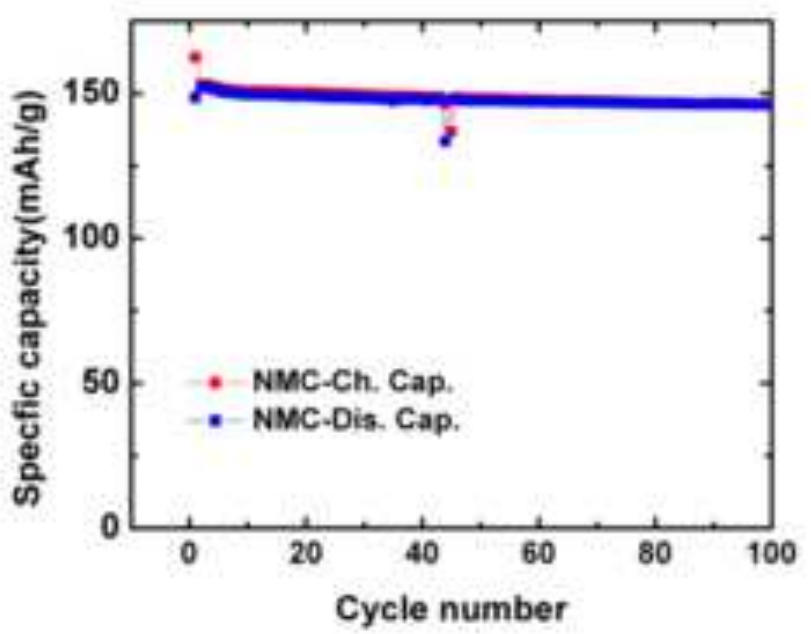



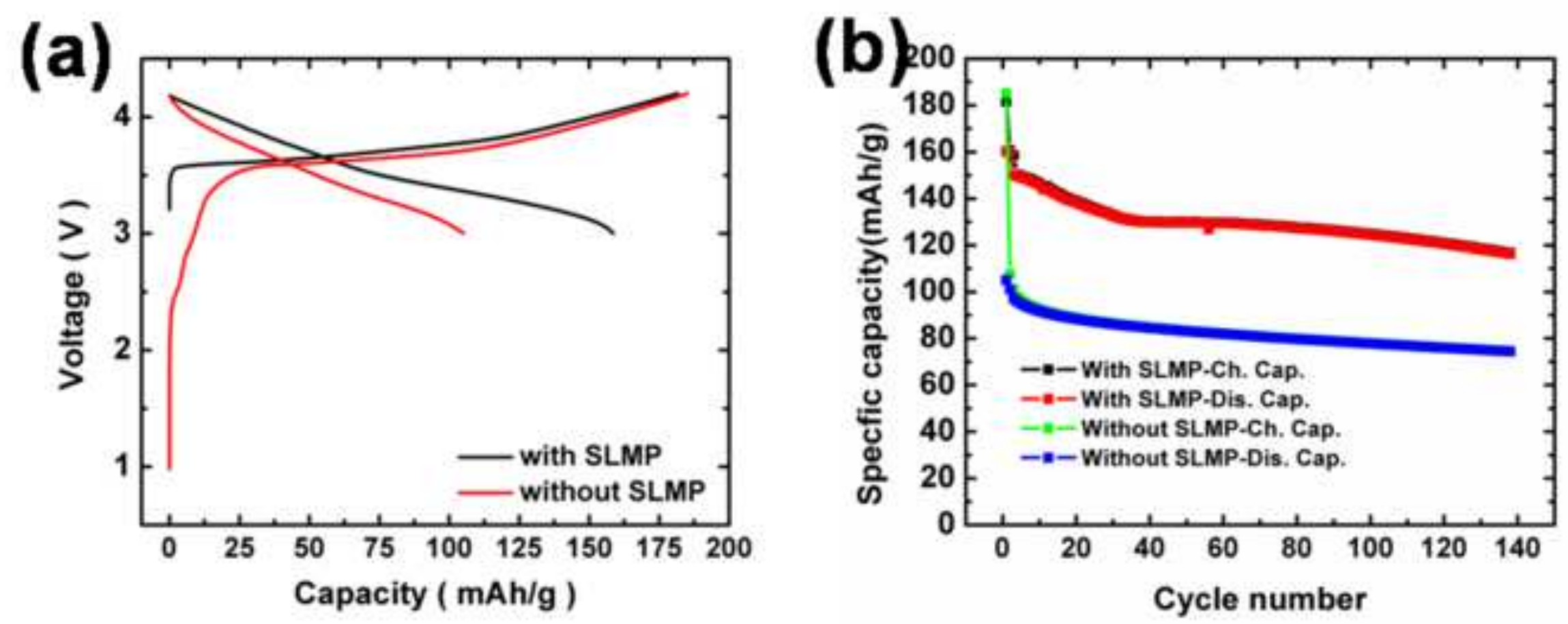


\section{Figure captions}

Fig. 1 Photo images of SLMP suspension in toluene and xylene without addition of polymer, rested for 0 second (a), 30 seconds (b), and 120 seconds (c).

Fig. 2 Photo images of SLMP suspension in 0\%, 0.5\%, 1\%, 3\%, and 5\% SBR binder solution, rested for 0 second (a), 30 seconds, (b) and 120 seconds (c). The SEM images of SLMP loading on a graphite surface with $0 \%(\mathrm{a}, \mathrm{b}, \mathrm{c}), 0.5 \%(\mathrm{~d}, \mathrm{e}, \mathrm{f}), 1 \%(\mathrm{~g}, \mathrm{~h}, \mathrm{i}), 3 \%(\mathrm{j}, \mathrm{k}, \mathrm{l})$, and $5 \%(\mathrm{~m}, \mathrm{n}, \mathrm{o})$ SBR binder solution. The scale bar for each row of SEM is the same and shown at the bottom.

Fig. 3 The SEM morphology of an SLMP-coated anode surface after pressure activation (a) (b) and after immersing in electrolyte for 48 hours (c) (d).

Fig. 4 The SEM images of SLMP loading on graphite surface before, after calendaring, and after immersing in electrolyte for 48 hours with $1 \%$ PS (a, d, g), 1\% SBR (b, e, h), and $0.5 \%$ PS with $0.5 \%$ SBR (c, f, i) binder solution.

Fig. 5 Photo images of SLMP suspension in $1 \%$ PS, $1 \%$ SBR, and $0.5 \%$ PS with $0.5 \%$ SBR binder solution, rested for 0 second (a), 30 seconds (b), and 120 seconds (c). The photo images of SLMP loading on a large piece of graphite anode surface with $1 \%$ PS (d), $1 \%$ SBR (e), and $0.5 \%$ PS with $0.5 \%$ SBR (f) binder solution.

Fig. 6 The voltage profile for the first cycle (a), and cycling performance (b) for a graphite/NMC full cell, the first-cycle voltage (c), and cycling performance (d) for graphite half cell and the first-cycle voltage (e), and cycling performance (f) NMC half cell. The performance of each cell both with and without SLMP prelithiation is plotted in (a-d).

Fig. 7 The voltage profile for the first-cycle (a) and cycling performance (b) for SiO/ NMC full cell. 